\author{
Fernanda Veruska Narciso ${ }^{1,3}$ \\ Cristiane Westin Teixeira ${ }^{1}$ \\ Luciana Oliveira e Silva ${ }^{1,3}$ \\ Renata Guedes Koyama ${ }^{1}$ \\ Adriana Neves da Silva Carvalho ${ }^{1}$ \\ Andrea Maculano Esteves ${ }^{2}$ \\ Sérgio Tufik ${ }^{1,3}$ \\ Marco Túlio de Mello ${ }^{1,3}$
}

\section{Maquinistas ferroviários: trabalho em turnos e repercussões na saúde}

\author{
Train drivers: shiftwork and health impacts
}

\author{
${ }^{1}$ Associação Fundo de Incentivo a \\ Pesquisa, Centro Multidisciplinar em \\ Sonolência e Acidentes. São Paulo, SP, \\ Brasil. \\ ${ }^{2}$ Universidade Estadual de Campinas, \\ Faculdade de Ciências Aplicadas. \\ Limeira, SP, Brasil. \\ ${ }^{3}$ Universidade Federal de São Paulo, \\ Departamento de Psicobiologia. São \\ Paulo, SP, Brasil. \\ Contato: \\ Marco Túlio de Mello \\ E-mail: \\ tmello@demello.net.br
}

O presente estudo foi apresentado no Congresso Nacional do Sono, realizado em Belo Horizonte-MG, em 2011, e seu resumo foi publicado em anais: Teixeira, C. W. et al. Sociodemographic and Health Profile of Brazilian Railway Shift Workers. Sleep Science, v. 4, n. 4, p. s131-s181, 2011.

A pesquisa recebeu apoio (espaço físico e realização de exames) da Associação Fundo de Incentivo à Pesquisa e do Centro Multidisciplinar em Sonolência e Acidentes.

Os autores declaram não haver conflitos de interesses.

\section{Resumo}

Objetivo: descrever o impacto do trabalho em turnos na saúde, no sono e na qualidade de vida de maquinistas ferroviários. Métodos: foram avaliados 611 maquinistas (escalas de trabalho $4 \times 1$ e $6 \times 2$ ), por meio de polissonografia, Índice da Qualidade do Sono de Pittsburgh, Índice de Gravidade de Insônia, Questionário de Qualidade de Vida SF-36, Questionário de Horne e Östberg, Capacidade de Trabalho e Escala de Sonolência de Epworth. Resultados: os maquinistas apresentaram idade média de 36,6 \pm 15,1 anos, 22\% apresentaram obesidade e $38,1 \%$ risco para doenças cardiovasculares. Em relação ao sono, $64,2 \%$ dos maquinistas relataram qualidade ruim de sono, $11,6 \%$ apresentaram distúrbios do sono e 29,3\% sonolência excessiva. Os resultados da polissonografia mostraram que $36,1 \%$ deles apresentaram apneia do sono e $47,2 \%$ demonstraram eficiência do sono reduzida. Além disso, os maquinistas apresentaram baixos índices de qualidade de vida, especialmente os da escala $4 \times 1$. Conclusão: é possível afirmar, na população estudada, que a exposição ao trabalho em turnos, a rotatividade inversa, pouco tempo dispensado às folgas e horas extras de trabalho estão associados a danos ao bem-estar, saúde, sociabilização e ao sono de qualidade desses trabalhadores e que provavelmente os fatores relacionados aos turnos de trabalho contribuem para esses danos.

Palavras-chave: sono; saúde; qualidade de vida; maquinistas; trabalho em turnos.

\begin{abstract}
Objective: to describe the impact of shiftwork on the health, sleep and quality of life of railway drivers. Methods: six hundred eleven railway drivers (schedule $4 \times 1$ and 6x2) were evaluated by polysomnography, Pittsburgh Sleep Quality Index, Insomnia Severity Index, Work Capacity Index, Quality of Life questionnaire (SF-36), Morningness-eveningness questionnaire (MEQ), and Epworth Sleepiness Scale. Results: railway drivers assessed were $36.6 \pm 15.1$ years of age, $22 \%$ were obese, and $38.1 \%$ presented risks for cardiovascular disease. Poor sleep quality $64.2 \%, 11.6 \%$ had sleep disorders and $29.3 \%$ excessive sleepiness. The polysomnography results showed that $36.1 \%$ of them presented sleep apnea and $47.2 \%$ had significant reduction in sleep efficiency. The railway drivers showed low levels of quality of life, especially those working under the $4 \times 1$ schedule. Conclusion: it is possible to assert that exposure to shiftwork, reverse rotation schedule, few days off, and working overtime are associated with impairment to well-being, health, sociability and workers' sleep quality, and the factors related to shiftwork probably contribute to these findings.
\end{abstract}

Keywords: sleep; health; quality of life; train drivers; shiftwork. 


\section{Introdução}

O setor ferroviário representa uma importante atividade socioeconômica em vários países e tem sido foco de pesquisas de avaliação relativas ao impacto do trabalho em turnos e noturno na vida desses trabalhadores (HÄRMÄ et al., 2002; KOYAMA et al., 2012).

Os trabalhadores em turnos, rotineiramente, queixam-se de distúrbios do sono, fadiga e sonolência durante a jornada de trabalho, o que tem sido considerado um importante fator de erros e risco de acidentes (ÅKERSTEDT; WRIGHT, 2009; WAGSTAFF; SIGSTAD LIE, 2011).

No estudo de Härmä et al. (2002), mais da metade dos maquinistas e controladores de tráfego ferroviário avaliados relatou fadiga grave durante o turno noturno. Além disso, o baixo desempenho devido à fadiga foi relatado por 21,0 a $37,0 \%$ dos maquinistas e 13,0 a $19,0 \%$ dos controladores.

O estudo de Folkard e Tucker (2003) demonstrou que há redução da segurança e da produtividade durante o turno noturno de trabalho. E essa redução é refletida por diversos fatores como dessincronização do ritmo circadiano, problemas de saúde, distúrbios do sono e prejuízos nas relações sociais.

Diversos estudos demonstraram a alta incidência e prevalência de distúrbios do sono em trabalhadores em turnos (LOPES et al., 2008; KOYAMA et al., 2012). Entre os mais de 80 distúrbios classificados pela American Academy of Sleep Medicine (2005), a Síndrome da Apneia Obstrutiva do Sono (SAOS) é o distúrbio mais comum entre os trabalhadores em turnos e noturno.

Nena et al. (2008) encontraram elevados índices de obesidade e SAOS em maquinistas gregos comparados à população em geral. No Brasil, a prevalência de SAOS na população da cidade de São Paulo é de 32,9\% (TUFIK et al., 2010). A SAOS está associada a constantes dessaturações da oxi-hemoglobina e resulta em fragmentação do sono e aumento do número de despertares devido ao esforço respiratório e, muitas vezes, é acompanhada de sonolência excessiva (GURUBHAGAVATULA, 2010; MANNARINO; DI FILIPPO; PIRRO, 2012).

Para Buysse et al. (2010) e Grandner et al. (2010), dormir pouco está relacionado às doenças cardiovasculares, câncer, acidente vascular cerebral, distúrbios gastrintestinais, diabetes, hipertensão arterial, além de depressão e distúrbios do sono.

Ku e Smith (2010) relataram que uma das principais causas de acidentes ferroviários é a fadiga relacionada às escalas de trabalho e aos fatores organizacionais, visto que existe uma influência da fadiga e da escala de trabalho no bem-estar, saúde e qualidade de vida dos ferroviários.

Nesse contexto, torna-se importante analisar fatores relacionados à saúde e bem-estar que são passíveis de influenciar a qualidade de vida e o desempenho no trabalho de trabalhadores em turnos, especialmente os maquinistas ferroviários. Dessa forma, o objetivo do presente estudo foi descrever o impacto do trabalho em turnos na saúde, no sono e na qualidade de vida de maquinistas ferroviários brasileiros.

\section{Métodos}

\section{Amostra}

O presente estudo foi realizado com uma amostra de maquinistas ferroviários de uma empresa de mineração, avaliados entre fevereiro de 2004 e fevereiro de 2005. De 712 maquinistas convidados a participar do estudo, 48 se recusaram a participar, 10 não assinaram o Termo de Consentimento Livre e Esclarecido e 43 deles não completaram todas as avaliações. Dessa forma, a amostra consistiu de 611 maquinistas de trem e manobristas de trem de pátio, todos do gênero masculino, que trabalhavam em turnos, em escalas rotativas $4 \times 1$ e $6 \times 2$.

A escala 4 x 1 é uma escala rotativa inversa destinada aos maquinistas que trabalhavam exclusivamente com manobras de trens de pátio, sendo quatro dias consecutivos de trabalho (jornadas com duração de 8 horas) e um dia de folga (duração de 24 horas). A escala 6 × 2 é uma escala rotativa direta realizada por maquinistas que trabalhavam exclusivamente nos deslocamentos dos trens (viagens), sendo seis dias consecutivos de trabalho (jornadas com duração de seis horas) e dois dias de folga (duração de 48 horas). A escala de rotação direta ou de sentido horário acompanha o sentido da rotação do relógio, ou seja, os horários se modificam da seguinte forma: turno matutino, vespertino e noturno. Já na escala de rotação inversa ou de sentido anti-horário os horários se modificam nesse sentido: turno noturno, vespertino e matutino.

Todos os maquinistas foram informados sobre os procedimentos do estudo e assinaram o Termo de Consentimento Livre e Esclarecido. O Comitê de Ética da Universidade Federal de São Paulo aprovou o protocolo do presente estudo (n. 1597-1503).

\section{Desenho do estudo, instrumentos e técnicas de coleta de dados}

Este estudo observacional e transversal abrangeu maquinistas, trabalhadores em turnos, de uma empresa de mineração que realiza suas atividades no Brasil. 
Ao todo, foram avaliados 381 maquinistas da escala $6 \times 2$ e 230 maquinistas da escala $4 \times 1$, assim como foram comparadas as escalas de trabalho desses trabalhadores. Para tanto, os maquinistas foram convidados a comparecer ao laboratório do sono, montado em um hotel, usualmente utilizado para descanso entre as jornadas de trabalho.

Todos os maquinistas realizaram individualmente o exame de polissonografia (PSG) para avaliar o padrão de sono. A massa corporal, estatura e circunferências da cintura, quadril e pescoço foram coletadas para determinar o índice de massa corporal (IMC) e o risco para doenças cardiovasculares. Ademais, todos os participantes responderam os questionários gerais para avaliação dos aspectos sociodemográficos, do estilo de vida e da rotina de trabalho. Questionários específicos foram utilizados na avaliação do padrão e qualidade do sono, qualidade de vida e capacidade de trabalho. Os instrumentos e técnicas de coleta são descritos a seguir.

\section{Exame de Polissonografia (PSG)}

A PSG foi realizada no período da noite, após a jornada de trabalho do turno da tarde (segundo dia de jornada), o mais próximo possível do horário habitual de sono de cada maquinista.

Para a realização da PSG foi utilizado o sistema digital portátil Embla ${ }^{\circledR}$ titanium. O exame foi realizado de acordo com Rechtschaffen e Kales (1968) e os eletrodos foram colocados de acordo com o sistema 10-20 (JASPER, 1958; IBER et al., 2007). A sala utilizada para a gravação do exame tinha uma cama confortável, luz e temperatura controladas. Os seguintes canais foram incluídos: EEG (C3-A2, C4-A1, O2-A1), EOG e EMG de tibial anterior e do queixo, ECG, fluxo de ar (sensor térmico), cinta tóraco-abdominal para movimentos respiratórios, microfone na lateral do pescoço para detectar o ronco, oximetria de pulso e um sensor de posição do corpo. As 32 épocas do sono foram estageadas de acordo com critérios padronizados e visualmente inspecionadas pelo especialista do sono. Os seguintes parâmetros foram analisados: (a) o Tempo Total de Sono (TTS, em minutos), definido como o tempo de duração do sono, (b) a latência do sono (em minutos), definido como o tempo de luz apagada até o início de 3 épocas consecutivas de fase 1 do sono de ondas lentas ou sono mais profundo, (c) a eficiência do sono, definida como a porcentagem do tempo total de duração do sono durante a gravação do exame, (d) tempo de vigília após o início do sono (Waso, em minutos), definido como o tempo total entre a vigília pontuada como o início do sono e despertar final, (e) as fases 1, 2 ,3 e REM do sono, assim como a porcentagem de TTS e, (f) a latência do sono REM, definida como o tempo desde o início do sono até a primeira época de sono REM. O diagnóstico de SAOS e o Índice de Apneia-Hipopneia (IAH) foram estabelecidos de acordo com os padrões da American Academy of Sleep Medicine (2005). A gravidade SAOS é definida como leve para IAH $\geq 5$ e $<15$, moderado para $\mathrm{IAH} \geq 15$ e $\leq 30$ e grave para $\mathrm{IAH}>30 /$ hora (IBER et al., 2007).

\section{Índice da Qualidade do Sono de Pittsburgh (IQSP)}

O IQSP foi utilizado como instrumento para avaliação subjetiva da qualidade do sono (CEOLIM; MENNA-BARRETO, 2000). O questionário é composto por 19 itens pontuados em uma escala de 0 a 3. Os itens são alocados em sete grupos, sendo: (1) qualidade subjetiva do sono; (2) latência do sono; (3) duração do sono; (4) eficiência habitual do sono; (5) alterações do sono; (6) uso de medicações do sono; (7) disfunção diurna. A partir do somatório dos escores dos sete grupos é determinado o IQSP. O resultado do IQSP varia entre 0 e 21 , sendo classificado respectivamente como qualidade de sono boa (0 a 4), qualidade de sono ruim (5 a 10) e indicação de distúrbio do sono (acima de 10).

\section{Índice de Gravidade de Insônia (IGI)}

Escala autoaplicável, especificamente desenvolvida para a avaliação da percepção do paciente em relação a sua insônia. Avalia especificamente os sintomas subjetivos da insônia, bem como o grau de preocupação e as dificuldades geradas ao paciente. É composta por sete itens classificados em uma escala entre 0 e 4 cujo somatório determina a classificação da gravidade de insônia como não significativa (entre 0 e 7), limite inferior (entre 8 e 14), moderada (entre 15 e 21) e grave (acima de 22) (BASTIEN; VALLIÈRES; MORIN, 2001).

\section{Questionário de Qualidade de Vida (SF-36)}

O questionário SF-36 é de fácil administração e compreensão para a avaliação subjetiva da qualidade de vida. Trata-se de um instrumento multidimensional composto por 36 itens que avaliam oito fatores: capacidade funcional (10 itens); aspecto físico (quatro itens); dor (dois itens); estado geral de saúde (cinco itens); vitalidade (quatro itens); aspectos sociais (dois itens); aspectos emocionais (três itens); saúde mental (cinco itens) e uma questão para a avaliação comparativa entre as condições de saúde atual e de um ano atrás. Para avaliar os resultados, é determinado um escore para cada uma das questões que, posteriormente, são transformadas em escalas de 
0 a 100, nas quais 0 corresponde a um pior estado de saúde e 100 a um melhor estado, onde cada fator é analisado separadamente (CICONELLI et al., 1999).

\section{Escala de Sonolência de Epworth (ESE)}

Atualmente, Epworth é a escala mais utilizada para a avaliação subjetiva da sonolência diurna e é capaz de diferenciar as pessoas com e sem sonolência daquelas com sonolência excessiva. Ela consiste em oito questões que descrevem situações cotidianas que podem induzir à sonolência. Cada questão é graduada de 0 a 3 pontos, sendo que escores acima de 10 indicam sonolência diurna significativa e acima de 15 estão associados à sonolência patológica presente em condições específicas, tais como apneia obstrutiva do sono e narcolepsia (JOHNS, 1991).

\section{Questionário de Horne e Östberg (HO)}

Questionário autoaplicável que foi utilizado para caracterizar a matutinidade/vespertinidade dos trabalhadores. Ele é amplamente utilizado nos estudos do ciclo vigília-sono e composto dos seguintes critérios: horários preferenciais de acordar e dormir; horários de maior disposição para atividades físicas e intelectuais; grau de dificuldade com que a pessoa executa determinadas tarefas em determinados horários e a autoclassificação da pessoa em um dos cinco tipos de cronotipo (matutino, matutino moderado, indiferente, vespertino moderado e vespertino) (HORNE; OSTBERG, 1976; BENEDITO-SILVA et al., 1990).

\section{Índice de Capacidade de Trabalho (ICT)}

Um dos métodos de autoavaliação realizado para determinar-se a capacidade do trabalho dos maquinistas foi o Índice de Capacidade de Trabalho. O ICT diagnostica de forma confiável as mudanças na capacidade para o trabalho nos diferentes grupos ocupacionais. Ele mostra efetividade em razão do acelerado envelhecimento da população e das consequências negativas observadas tanto na inserção quanto na manutenção, assim como nas condições de saúde dos brasileiros com mais de 30 anos (TUOMI et al., 2005).

\section{Análise estatística}

A análise estatística foi realizada por meio do Statistical Software Package (SPSS Statistics for Windows, versão 18.0; SPSS Inc., Chicago, IL). O teste de Kolmogorov-Smirnov foi utilizado para avaliar a normalidade dos dados. As variáveis com distribuição não normal foram transformadas usando um Z-score.
Na estatística descritiva, os dados contínuos foram apresentados em média \pm DP (desvio padrão) e, os categóricos, demonstrados por meio da frequência relativa $(\%)$.

Para a comparação das variáveis de natureza contínua (gerais, antropométricas, cronotipo e de trabalho) entre os grupos, foi utilizado o teste de Análise de Variância (ANOVA). A comparação entre os parâmetros do sono e os questionários entre as escalas de trabalho ( $4 \times 1$ e $6 \times 2$ ) foi realizada pela Análise de Covariância (ANCOVA) com o ajuste para as variáveis confundidoras: idade, IMC e IAH. O teste de ANCOVA foi utilizado também na comparação da qualidade de vida entre as escalas de trabalho, ajustado para as variáveis confundidoras: idade, IMC, IAH e anos de escolaridade.

Para a comparação das variáveis categóricas foi utilizado o teste qui-quadrado de Pearson. O nível de significância considerado foi de $5 \%$.

\section{Resultados}

Na Tabela 1 são mostradas as características gerais, antropométricas, de trabalho e de sono dos 611 maquinistas avaliados nas duas escalas $6 \times 2$ e $4 \times 1$. A média etária da amostra foi de $36,6 \pm 15,1$ anos, sendo os maquinistas da escala $6 \times 2$ mais velhos (38,6 $\pm 17,6$ anos). Ao comparar os grupos, vimos que os maquinistas da escala $6 \times 2$ eram de cronotipo matutino $(62,5 \%)$, apresentavam maior escolaridade, tinham mais tempo de trabalho em turnos (14,5 anos) e a maioria deles realizava horas extras (96,3\%). Já os maquinistas da escala $4 \times 1$ tinham 9,4 anos de trabalho em turnos, 41,1\% apresentavam cronotipo indiferente, $13,6 \%$ realizavam horas extras de trabalho e gastavam mais tempo de deslocamento do trabalhoresidência (41,1 $\pm 22,6$ minutos), quando comparados aos da escala 6 × 2 (24,1 $\pm 24,4$ minutos). A maioria dos maquinistas de ambas as escalas $(64,6 \%)$ era de casados e tinha filhos dependentes (75,5\%).

Nas avaliações do IMC, das circunferências da cintura e do pescoço dos maquinistas foram observados fatores de risco para a obesidade, doenças cardiovasculares e para a apneia obstrutiva do sono (Tabelas 1 e 2).

No geral, 69,5\% da amostra apresentaram sobrepeso $(69,5 \%)$ e obesidade $(22,0 \%) ; 13,0 \%$ eram fumantes, $33,6 \%$ ingeriam bebidas alcoólicas e 38,1\% apresentavam risco de doenças cardiovasculares (dados não apresentados em tabela). Diante disso, os maquinistas da escala 6 × 2 fumavam mais, tinham maior IMC e ganharam mais massa corporal em 6 meses. Contudo, os maquinistas da escala 4 x 1 apresentaram maior circunferência da cintura e do pescoço. 
Tabela 1 Comparação de grupos de trabalho em turnos dos maquinistas ferroviários da empresa estudada, segundo características sociodemográficas, antropométricas, de trabalho, de cronotipo e prevalência de uso de tabaco e álcool (2004-2005)

\begin{tabular}{|c|c|c|c|c|c|}
\hline Características & $\begin{array}{l}\text { Amostra total } \\
(n=611)\end{array}$ & $\begin{array}{c}\text { Escala } \\
6 \times 2 \\
(n=381)\end{array}$ & $\begin{array}{c}\text { Escala } \\
4 \times 1 \\
(n=230)\end{array}$ & $F$ & $p$ \\
\hline \multicolumn{6}{|l|}{ Sociodemográficas } \\
\hline Idade - anos completos* & $36,6(15,1)$ & $38,6(17,6)$ & $33,4(8,5)$ & 17,3 & $<0,0001^{\dagger}$ \\
\hline \multicolumn{6}{|l|}{ Estado civil (\%) } \\
\hline Solteiros & 17,9 & 11,8 & 21,8 & & \\
\hline Casados & 64,6 & 64,8 & 48,6 & 23,6 & $<0,001^{\dagger}$ \\
\hline Outros & 17,5 & 23,4 & 29,6 & & \\
\hline Filhos dependentes $(\%)^{\ddagger}$ & 75,5 & 72,2 & 48,9 & 38,2 & $0,001^{\dagger}$ \\
\hline Anos de escolaridade* & $11,9(2,0)$ & $12,0(2,0)$ & $11,6(2,0)$ & 5,2 & $0,02^{\dagger}$ \\
\hline \multicolumn{6}{|l|}{ Antropométricas } \\
\hline $\mathrm{IMC}\left(\mathrm{kg} / \mathrm{m}^{2}\right)^{*}$ & $26,8(4,1)$ & $27,2(4,3)$ & $26,1(3,6)$ & 11,0 & $0,001^{\dagger}$ \\
\hline Circunferência da cintura $(\mathrm{cm})^{*}$ & $91,6(9,9)$ & $97,2(66,3)$ & $101,9(103,8)$ & 0,46 & 0,49 \\
\hline Circunferência do pescoço $(\mathrm{cm})^{*}$ & $38,9(3,4)$ & $44,5(69,5)$ & $50,4(109,3)$ & 0,67 & 0,41 \\
\hline Ganho de peso último semestre $(\%)^{\ddagger}$ & 40,4 & 39,4 & 26,1 & 9,12 & $0,003^{\dagger}$ \\
\hline \multicolumn{6}{|l|}{ Trabalho } \\
\hline Tempo de trabalho em turno (anos)* & $12,6(8,5)$ & $14,5(8,8)$ & $9,4(6,9)$ & 49,0 & $<0,001^{\dagger}$ \\
\hline $\begin{array}{l}\text { Tempo de deslocamento trabalho- } \\
\text { residência (minutos)* }\end{array}$ & $30,9(25,2)$ & $24,1(24,4)$ & $41,1(22,6)$ & 74,5 & $<0,0001^{\dagger}$ \\
\hline Hora extra $(\%)^{\ddagger}$ & 61,2 & 96,3 & 13,6 & 419,0 & $<0,0001^{\dagger}$ \\
\hline $\mathrm{ICT}^{*}$ & $41,9(5,6)$ & $40,9(5,6)$ & $43,6(5,1)$ & 32,9 & $<0,001^{\dagger}$ \\
\hline Ótima $(\%)^{\ddagger}$ & 48,5 & 41,5 & 46,8 & & \\
\hline Boa $(\%)^{\ddagger}$ & 30,7 & 29,6 & 25,0 & & \\
\hline Moderada $(\%)^{\ddagger}$ & 20,3 & 28,0 & 27,5 & & \\
\hline Baixa $(\%)^{\frac{1}{2}}$ & 0,5 & 0,8 & 0,7 & & \\
\hline \multicolumn{6}{|l|}{ Cronotipo } \\
\hline Cronotipo (média geral)* & $58,6(9,4)$ & $60,0(9,3)$ & $56,1(9,1)$ & 25,0 & $<0,0001^{\dagger}$ \\
\hline Matutino $(\%)^{\ddagger}$ & 55,1 & 62,5 & 31,4 & & \\
\hline Indiferente $(\%)^{\ddagger}$ & 40,7 & 33,1 & 41,1 & & \\
\hline Vespertino $(\%)^{\ddagger}$ & 4,2 & 4,4 & 27,5 & & \\
\hline \multicolumn{6}{|l|}{ Tabaco e álcool } \\
\hline Fumantes $(\%)^{\ddagger}$ & 13,0 & 13,4 & 7,9 & & \\
\hline Uso de álcool > 3x/semana (\%) & 33,6 & 47,0 & 9,3 & & \\
\hline
\end{tabular}

* Dados apresentados em média (desvio padrão)

† Diferença estatística ao comparar os grupos por meio do teste ANOVA, $p<0,05$

¥ Dados apresentados em frequência relativa (\%): Teste Qui Quadrado de Pearson

IMC: Índice de Massa Corporal; ICT: Índice de Capacidade de Trabalho; Cronotipo: Avaliado por meio do Questionário de Horne e Östberg

Em relação à avaliação subjetiva do sono, 64,2\% da amostra total relataram qualidade ruim de sono e 11,6\% apresentaram distúrbios do sono (Tabela 3). Observou-se nos valores médios da arquitetura do sono (avaliação por meio da PSG), dos maquinistas de ambas as escalas de trabalho, que $36,1 \%$ deles apresentaram SAOS (Tabela 2). E, ainda, $47,2 \%$ da amostra total mostraram eficiência do sono reduzida e
29,3\% sonolência excessiva (dados não apresentados em tabela).

Ao comparar os resultados do padrão de sono aos valores de referência descritos por Carskadon e Dement (1994), verificamos que a latência do sono REM apresentou-se reduzida em $25,9 \%$ de toda a amostra, o N1 estava reduzido em $39,0 \%$ e aumentado em $16,7 \%$ dos maquinistas, $34,4 \%$ apresentaram N2 
Tabela 2 Parâmetros da arquitetura do sono aferidos por polissonografia nos maquinistas ferroviários amostra total e comparação entre os grupos de estudo (escala $6 \times 2$ e escala $4 \times 1$ )

\begin{tabular}{|c|c|c|c|c|c|}
\hline Arquitetura do sono & $\begin{array}{c}\text { Amostra total } \\
\quad(n=611)\end{array}$ & $\begin{array}{c}\text { Escala } \\
6 \times 2 \\
(n=381)\end{array}$ & $\begin{array}{c}\text { Escala } \\
4 \times 1 \\
(n=230)\end{array}$ & F & $p$ \\
\hline Eficiência* & $81,9(12,7)$ & $81,1(15,9)$ & $83,4(20,6)$ & 4,74 & $0,03^{\dagger}$ \\
\hline Tempo Total de Sono (TTS)* & $357,2(75,3)$ & $361,1(95,5)$ & $345,9(124,0)$ & 5,45 & $0,02^{\dagger}$ \\
\hline Latência sono** & $22,7(26,0)$ & $24,9(33,5)$ & $18,8(42,8)$ & 2,29 & $0,007^{\dagger}$ \\
\hline Latência REM* & $109,3(62,1)$ & $116,9(78,7)$ & $98,4(102,1)$ & 11,81 & $0,001^{\dagger}$ \\
\hline$\% \mathrm{~N} 1^{*}$ & $3,4(5,1)$ & $2,25(5,7)$ & $5,1(7,4)$ & 52,52 & $<0,0001^{\dagger}$ \\
\hline$\% \mathrm{~N}^{*}$ & $48,8(21,0)$ & $50,8(26,9)$ & $45,6(34,9)$ & 8,01 & $0,005^{\dagger}$ \\
\hline$\% \mathrm{~N}^{*}$ & $26,7(8,4)$ & $26,7(10,4)$ & $26,9(13,5)$ & 0,16 & 0,68 \\
\hline$\%$ Sono REM" & $20,2(7,5)$ & $20,0(8,0)$ & $20,6(6,6)$ & 2,82 & 0,63 \\
\hline Mínimo de vigília* & $53,7(43,3)$ & $56,7(54,6)$ & $49,3(70,8)$ & 3,96 & $0,04^{\dagger}$ \\
\hline Índice desp/h* & $11,3(8,1)$ & $10,5(8,1)$ & $12,6(10,5)$ & 15,1 & $<0,0001^{\dagger}$ \\
\hline SAOS IAH $\geq 5(\%)^{\ddagger}$ & 36,1 & 36,1 & 34,1 & 0,25 & 0,61 \\
\hline
\end{tabular}

N2: Estágio 2 do sono de ondas lentas; N3: Estágio 3 do sono de ondas lentas; Índice desp/h: Índice de despertares por hora; IAH: Índice de Apneia e Hipopneia; SAOS: Síndrome Apneia Obstrutiva do Sono

* Dados apresentados em média (DP) - ANCOVA com ajuste de idade, IMC e IAH

† Diferença estatística ao comparar os grupos por meio do teste ANCOVA, ajustado por idade, IMC e IAH, p $\leq 0,05$

\#ados apresentados em frequência relativa (\%)

Tabela 3 Comparação de grupos de trabalho em turnos dos maquinistas ferroviários da empresa estudada, segundo a avaliação subjetiva do sono

\begin{tabular}{lccccc}
\hline \multicolumn{1}{c}{ Avaliação subjetiva do sono } & $\begin{array}{c}\text { Amostra total } \\
(n=611)\end{array}$ & $\begin{array}{c}\text { Escala } \\
6 \times 2 \\
(n=381)\end{array}$ & $\begin{array}{c}\text { Escala } \\
4 \times 1 \\
(n=230)\end{array}$ & $F$ & $p$ \\
\hline Epworth* $^{\text {Pittsburgh" }}$ & $7,7(3,5)$ & $7,9(4,5)$ & $7,6(5,8)$ & 0,95 & 0,32 \\
Qualidade boa (\%) & $6,8(3,0)$ & $6,1(3,6)$ & $7,9(4,8)$ & 55,82 & $<0,0001^{\dagger}$ \\
Qualidade ruim (\%) & 24,2 & & & & \\
Distúrbio do sono (\%) & 64,2 & & & & \\
Insônia* & 11,6 & & & & \\
\hline
\end{tabular}

* Dados apresentados em média (DP)

† Diferença estatística ao comparar os grupos por meio do teste ANCOVA, ajustado por idade, IMC e IAH: $\mathrm{p} \leq 0,05$

* Dados representados em frequência relativa (\%)

reduzido e 21,9\% aumentado, 67,0\% deles estavam com o estágio N3 aumentado e 42,4\% do estágio REM apresentaram-se reduzido. SAOS foi diagnosticada em $36,1 \%$ dos maquinistas, destes, $53,7 \%$ apresentaram SAOS leve, 26,4\% moderada e 19,9\% grave.

Ao comparar a arquitetura do sono entre os maquinistas pertencentes às escalas de trabalho $4 \times 1 \mathrm{e}$ $6 \times 2$, houve redução significativa do TTS, da latência para o início do sono e para início do sono REM, assim como do estágio N2 do sono dos maquinistas da escala $4 \times 1$, ajustado pelos fatores confundidores: idade, IAH e IMC. E, ainda, houve maior presença de insônia e um aumento do número de despertares durante o sono dos maquinistas da escala $4 \times 1$. Porém, os maquinistas da escala $6 \times 2$ apresentaram redução significativa da eficiência do sono $(81,1 \pm 15,9)$ em comparação aos maquinistas da escala $4 \times 1(83,4 \pm 20,6)$.

A Tabela 4 mostra os valores médios da qualidade de vida da amostra, sendo que o domínio vitalidade apresentou baixo escore na amostra total. Contudo, os maquinistas pertencentes à escala $4 \times 1$ apresentaram um maior comprometimento dos domínios do questionário de qualidade de vida, com diferença estatisticamente significativa $(\mathrm{p} \leq 0,05)$. 
Tabela 4 Comparação de grupos de trabalho em turnos dos maquinistas ferroviários da empresa estudada, segundo os escores dos domínios do questionário SF-36 (qualidade de vida)

\begin{tabular}{|c|c|c|c|c|c|}
\hline Domínios & $\begin{array}{l}\text { Amostra total } \\
\quad(n=611)\end{array}$ & $\begin{array}{c}\text { Escala } \\
6 \times 2 \\
(n=381)\end{array}$ & $\begin{array}{c}\text { Escala } \\
4 \times 1 \\
(n=230)\end{array}$ & $F$ & $p$ \\
\hline Total* & $75,9(25,7)$ & $78,2(27,3)$ & $72,1(22,3)$ & 226,94 & $<0,0001^{\dagger}$ \\
\hline Capacidade funcional* & $88,8(15,1)$ & $88,5(15,4)$ & $89,4(14,7)$ & 0,02 & 0,87 \\
\hline Aspectos físicos* & $89,3(21,5)$ & $91,5(18,4)$ & $85,6(26,0)$ & 10,22 & $0,001^{\dagger}$ \\
\hline Dor ${ }^{*}$ & $84,2(20,0)$ & $86,9(17,6)$ & $79,6(22,5)$ & 20,2 & $<0,0001^{\dagger}$ \\
\hline Estado de saúde geral* & $81,9(16,7)$ & $83,5(14,7)$ & $79,1(19,3)$ & 10,34 & $0,001^{\dagger}$ \\
\hline Vitalidade ${ }^{*}$ & $67,5(20,8)$ & $78,5(14,3)$ & $49,1(16,7)$ & 482,32 & $<0,0001^{\dagger}$ \\
\hline Aspectos sociais*" & $87,7(18,8)$ & $88,7(16,8)$ & $86,0(21,8)$ & 1,78 & 0,18 \\
\hline Aspectos emocionais* & $89,5(23,4)$ & $91,2(20,3)$ & $86,7(20,7)$ & 4,32 & $0,03^{\dagger}$ \\
\hline Saúde mental* & $76,0(17,3)$ & $83,4(14,5)$ & $63,7(14,6)$ & 226,94 & $<0,0001^{\dagger}$ \\
\hline
\end{tabular}

*Dados apresentados em média (DP)

† Diferença estatística ao comparar os grupos por meio do teste ANCOVA, ajustado por idade, IMC e IAH e anos de escolaridade, $\mathrm{p} \leq 0,05$

\section{Discussão}

No presente estudo, foi descrito o impacto do trabalho em turnos na saúde, no sono e na qualidade de vida de maquinistas ferroviários trabalhadores em turnos.

Em relação ao sono, os maquinistas apresentaram eficiência reduzida e qualidade ruim do sono, assim como distúrbios do sono. Desse modo, esses resultados podem estar relacionados à curta duração do sono prevalente entre os maquinistas do presente estudo. Adicionalmente, a arquitetura do sono dos maquinistas pertencentes à escala de trabalho $4 \times 1$ apresentou redução significativa do TTS, latência do sono REM, latência do sono e de N2, além de aumento do número de despertares e presença de insônia, comparados aos maquinistas da escala $6 \times 2$, apesar destes últimos terem apresentado menor eficiência do sono. Esses achados podem ser explicados pelo conhecido desajuste do ritmo biológico, causado pela inversão do ciclo vigília-sono, pelo acúmulo de débito de sono. No caso da escala 4 x 1, ocorre maior tempo de exposição ao trabalho (8 horas), maior número de deslocamentos trabalho-residência e, pelo fato de se ter somente um dia de folga, resta pouco tempo para o lazer, o convívio sociofamiliar e, principalmente, para o descanso restaurador (ÅKERSTEDT, 2003; OHAYON; SMOLENSKY; ROTH, 2010). E ainda, com o avançar da idade e maior tempo de exposição ao trabalho em turnos, há uma diminuição da qualidade do sono devido às alterações do ritmo da temperatura central, do cortisol e da melatonina, o que pode explicar a menor eficiência do sono relacionada aos maquinistas da escala 6 × 2 e às alterações no padrão de sono dos maquinistas da escala 4 x 1 (KRIPKE et al.,
2007; COSTA, 2010). Por outro lado, a presença de filhos dependentes pode influenciar na qualidade do sono e no tempo dispensado ao lazer e descanso (HÄRMÄ et al., 2002; OHAYON; SMOLENSKY; ROTH, 2010).

O estudo de Lopes et al. (2008) reportou que as alterações na qualidade do sono podem repercutir nos aspectos cognitivos e nas relações interpessoais. Do mesmo modo, o trabalho em turnos pode interferir nas atividades domésticas, na vida familiar e social (BARNES-FARRELL et al., 2008).

Além disso, a curta duração do sono, a presença de distúrbios do sono, o trabalho noturno e o excesso de horas trabalhadas podem alterar a arquitetura do sono, o ritmo vigília-sono, aumentar a fadiga física e mental, assim como afetar o estado de humor dos trabalhadores em turnos (HÄRMÄ et al., 2002; COSTA, 2010).

Similarmente, no estudo de Sallinen et al. (2003) houve uma diminuição da duração do TTS, especialmente antes do início do turno manhã e noite, assim como aumento da sonolência em maquinistas trabalhadores em turnos de escalas rotativas irregulares. O estudo epidemiológico de Ohayon, Smolensky e Roth (2010) com trabalhadores do estado de Nova Iorque (EUA) observou que a duração do sono principal entre os trabalhadores com escalas rotativas e noturnas foi inferior a 6,5 horas.

Além desses resultados, os dados da PSG do presente estudo mostraram uma prevalência de $36,1 \%$ e $34,1 \%$ de SAOS entre os maquinistas das escalas $6 \times 2$ e 4 × 1 , respectivamente, similar ao estudo epidemiológico realizado com a população da cidade de São Paulo $(32,9 \%)$ (TUFIK et al., 2010), porém apresentou-se mais elevada em relação a outros estudos realizados com 
motoristas (15,8\%), maquinistas (22\%) e trabalhadores em turnos (20\%) (HOWARD et al., 2004; NENA et al., 2008). Sabe-se que a incidência e prevalência dos distúrbios respiratórios do sono e do débito de sono estão intimamente relacionadas ao estilo de vida e às condições alteradas de saúde dos trabalhadores (HÄRMÄ et al., 2002; COSTA, 2010).

Nesse aspecto de saúde e estilo de vida, vimos que os maquinistas apresentaram altos índices de IMC, fumavam e grande parte deles ingeria bebidas alcoólicas. Semelhante ao presente estudo, Koyama et al. (2012) reportaram que 9,5\% dos maquinistas ferroviários avaliados eram fumantes, 54,7\% relataram uso de álcool e 77,0\% eram obesos $(27,7 \pm 4,4)$. Outros estudos que avaliaram motoristas e maquinistas apresentaram uma prevalência de $40,0 \%$ a $52,0 \%$ de excesso de peso, 20,0\% a 65,0\% de obesidade, 25,0\% a $60,0 \%$ de fumantes entre maquinistas e motoristas trabalhadores em turnos avaliados (MINA; CASOLIN, 2007; BENVEGNÚ et al., 2008; NENA et al., 2008). É conhecido na literatura que todas essas condições possibilitam a instalação de doenças físicas e mentais, assim como de distúrbios do sono, especialmente a SAOS.

Contudo, a literatura demonstra que a falta de sono pode provocar distúrbios hormonais e metabólicos. Por exemplo, a redução da sensibilidade à insulina, o aumento da grelina e a diminuição da leptina estão diretamente relacionados com a falta de sono e ganhos de massa corporal, contribuindo, assim, para a obesidade, distúrbios do sono e doenças cardiovasculares (MORGAN et al., 2003; SPIEGEL et al., 2004). No geral, os trabalhadores em turnos alteram a função circadiana metabólica, assim o horário e conteúdo das refeições tornam-se inapropriados e, dessa forma, há aumento da preferência por alimentos mais gordurosos, podendo levar às doenças como obesidade, diabetes e hipertensão arterial (COSTA, 2010).

Segundo Mina e Casolin (2007), existe uma relação entre os distúrbios respiratórios do sono, hipertensão e obesidade, fatores estes que podem maximizar o processo de fadiga, reduzir a qualidade de vida e aumentar o risco para os acidentes. Assim, Padilha et al. (2010) reportaram que trabalhadores em turnos apresentam alta propensão em desenvolver distúrbios metabólicos e obesidade. Por outro lado, alterações do padrão de sono e do estilo de vida são também consideradas como fatores de risco para aumento de massa corporal e de massa gordurosa, perda de massa magra e aparecimento de distúrbios do metabolismo da glicose e gordura (CRISPIM et al., 2007; NEDELTCHEVA et al., 2010).

Contudo, a obesidade, o fumo e a ingestão de bebida alcóolica prevalentes entre os trabalhadores em turnos podem estar associados com a alteração do ritmo vigília-sono, presença de sonolência e alterações na arquitetura do sono (KAGEYAMA et al., 2005; ZHANG et al., 2006), além de apresentarem risco para doenças crônicas não transmissíveis e contribuírem para redução do desempenho psicomotor e da qualidade de vida (COSTA, 2010; OLIVEIRA e SILVA et al., 2012).

Nesse contexto, os maquinistas da escala de trabalho 4 x 1 apresentaram baixo índice de qualidade de vida quando comparados aos maquinistas da escala $6 \times 2$, principalmente nos domínios dor, aspectos físicos, vitalidade, estado geral da saúde, aspectos emocionais e de saúde mental. Como visto, os escores de vitalidade e de saúde mental apresentaram-se ainda mais baixos, o que pode inferir um prejuízo no desempenho psicomotor e o desenvolvimento de cansaço e/ou fadiga nessa primeira amostra. Esses prejuízos da qualidade de vida dessa população podem estar diretamente relacionados à duração do turno, sentido de rotação da escala de trabalho (rotativo inverso), tempo gasto no deslocamento trabalho-residência, assim como pouco tempo livre dispensado com lazer, família e descanso durante a folga. A literatura mostra que esses fatores podem alterar os padrões de sono, induzir ou favorecer distúrbios metabólicos, diminuir o desempenho físico e mental, assim como interferir negativamente na vida pessoal e na tolerância ao trabalho em turnos (ÅKERSTEDT; WRIGHT, 2009; COSTA, 2010; ASAOKA et al., 2013).

Semelhante ao nosso estudo, Nena et al. (2008) avaliaram 226 maquinistas ferroviários gregos que apresentaram sobrepeso (28,7 $\left.\pm 3,7 \mathrm{~kg} / \mathrm{m}^{2}\right), 59,7 \%$ deles eram fumantes e $11,5 \%$ tinham apneia obstrutiva do sono. O índice de apneia e hipopneia do sono foi de 11-14 eventos por hora, 7,1\% deles relataram sonolência, os quais apresentaram escores do questionário SF-36 diminuídos nos domínios aspectos físicos e emocionais, dor e vitalidade, comparados com os maquinistas não sonolentos. No entanto, a qualidade de vida dos maquinistas em geral, assim como do presente estudo, não foi diferente da população grega e dos maquinistas brasileiros do estudo de Oliveira e Silva et al. (2012), exceto no aspecto vitalidade, no qual a nossa amostra apresentou baixos escores relevantes e diferentes das outras duas populações.

Ilmarinen, Tuomi e Klockars (1997) e Tuomi et al. (1997) relataram que o estilo de vida, as condições de saúde e características sociodemográficas são fatores que podem interferir na capacidade para o trabalho.

Para Chiu et al. (2007) e Milosevic et al. (2011), existe uma alta associação entre qualidade de vida e capacidade de trabalho em trabalhadores em turnos.

O estudo de Oliveira e Silva et al. (2012) reportou que baixa capacidade de trabalho, sobrepeso, ansiedade, depressão e sonolência apresentaram impacto negativo na qualidade de vida global de maquinistas, 
assim como as alterações no sono REM e de humor impactaram negativamente na capacidade funcional desses trabalhadores. Em relação à arquitetura do sono, o tempo total do sono REM dos maquinistas daquele estudo não diferiu da nossa amostra; entretanto, os maquinistas de ambas as escalas de trabalho do presente estudo demonstraram menor eficiência do sono. Sabe-se que essas condições de sono, saúde e os aspectos socioemocionais podem afetar a qualidade de vida, o que foi demonstrado no presente estudo com os maquinistas da escala 4 x 1 .

No entanto, em geral, os maquinistas da nossa amostra (48,5\%) referiram ótima capacidade de trabalho (41,9\%) similarmente aos estudos com trabalhadores em turnos de Metzner e Fischer (2001) e Oliveira e Silva et al. (2012). E, apesar dos maquinistas da escala $4 \times 1$ terem apresentado maior comprometimento dos domínios de qualidade de vida e redução da qualidade do sono, 46,8\% deles referiu ótima capacidade de trabalho. Entre as possíveis explicações para esse resultado encontrado, podemos destacar a presença do efeito do trabalhador saudável, que descreve um processo de seleção continuada de tal forma que aqueles que permanecem empregados tendem a ser mais saudáveis do que aqueles que deixam o emprego (ARRIGHI; HERTZ-PICCIOTTO, 1994; RICHARDSON et al., 2004). Como os maquinistas estudados já têm um tempo de exposição ao trabalho em turnos relativamente longos (9,4 \pm 6,9 anos) é possível que o ICT esteja refletindo tanto o efeito do trabalhador saudável quanto as prováveis estratégias de enfrentamento desenvolvidas por esses maquinistas em relação às condições do trabalho em turnos, como relatado por Metzner e Fischer (2001).

Portanto, observamos em nosso estudo que os maquinistas, em geral, especialmente os pertencentes à escala 4 x 1, apresentaram condições preocupantes de saúde e qualidade do sono, assim como maus hábitos de vida (fumo, uso de álcool, sobrepeso e obesidade) e condições ruins de trabalho (pouco tempo de folga e excesso de horas extras). Como bem relatado na literatura, a associação desses fatores pode levar a um aumento do cansaço, disfunção cognitiva, alterações emocionais e sono não restaurador, o que pode desencadear o processo de fadiga crônica e, consequentemente, uma possibilidade maior para a ocorrência de acidentes de trabalho.

As limitações do presente estudo compreendem a falta de um grupo controle de maquinistas que não trabalhavam em turnos, bem como o fato das avaliações não terem permitido comparações no período de férias dos trabalhadores alvo do estudo. Além disso, não foram obtidas informações sobre a renda mensal dos maquinistas, as quais poderiam ter influenciado as diferenças ocorridas nos domínios do questionário de qualidade de vida (SF-36). Outra limitação é que o estudo de corte transversal não permite estabelecer relação de causalidade, apenas associações, o que torna impossível afirmar de forma categórica que a rotatividade inversa, a exposição ao trabalho em turnos e pouco tempo para folgas apresentam maiores danos ao bem-estar, saúde e vida social na população estudada.

Entretanto, é possível afirmar, na população estudada, que a exposição ao trabalho em turnos, a rotatividade inversa, pouco tempo dispensado às folgas e horas extras de trabalho estão associados a maiores danos ao bem-estar, saúde, sociabilização e ao sono de qualidade e que provavelmente os fatores relacionados aos turnos de trabalho contribuem para esses danos.

Dessa forma, requerem-se mais estudos nessa área de transportes no Brasil, que abordem esses fatores relacionados à saúde mental e física do trabalhador, os quais podem estar associados a importantes prejuízos de natureza socioeconômica e de saúde pública.

\section{Contribuições de autoria}

Narciso, F. V. e Teixeira, C. W.: contribuíram de forma substancial no projeto, no levantamento de dados, na interpretação dos dados e na elaboração do manuscrito. Oliveira e Silva, L. e Koyama, R. G.: contribuíram de forma substancial na análise e interpretação dos dados. Carvalho, A. N. S.: contribuiu de forma substancial no levantamento dos dados e na elaboração do manuscrito. Esteves, A. M.: contribuiu de forma substancial na análise e interpretação dos dados do manuscrito, na revisão crítica e na elaboração final do mesmo. Mello, M. T. e Tufik, S.: contribuíram de forma substancial na revisão crítica e na aprovação final da versão publicada.

\section{Agradecimentos}

AFIP: Associação Fundo de Incentivo à Pesquisa; CEPE: Centro de Estudos em Psicobiologia e Exercício; CEMSA: Centro Multidisciplinar em Sonolência e Acidentes; CNPq: Conselho Nacional de Desenvolvimento Científico e Tecnológico. 


\section{Referências}

ÅKERSTEDT, T. Shift work and disturbed sleep/ wakefulness. Occupational Medicine, London, v. 53, n. 2, p. 89-94, 2003. http://dx.doi.org/10.1093/occmed/ kqg046. PMid:12637592

ÅKERSTEDT, T.; WRIGHT JUNIOR, K. P. Sleep loss and fatigue in shift work and shift work disorder. Sleep Medicine Clinics, New York, v. 4, n. 2, p. 257-271, 2009. http://dx.doi.org/10.1016/j. jsmc.2009.03.001. PMid:20640236

AMERICAN ACADEMY OF SLEEP MEDICINE AASM. The International classification of sleep disorders, revised: diagnostic and coding manual. Westchester: American Academy of Sleep Research; Philadelphia: WB Saunders Co., 2005.

ARRIGHI, H. M.; HERTZ-PICCIOTTO, I. The evolving concept of the healthy worker survivor effect. Epidemiology, Cambridge, v. 5, n. 2, p. 189-196, 1994. http://dx.doi.org/10.1097/00001648-199403000-00009. PMid:8172994

ASAOKA, S. et al. Factors associated with shift work disorder in nurses working with rapid-rotation schedules in Japan: the nurses' sleep health project. Chronobiology International, London, v. 30, n. 4, p. 628-636, 2013. http://dx.doi.org/10.3109/07420528.201 2.762010. PMid:23445510

BARNES-FARRELL, J. L. et al. What aspects of shiftwork influence off-shift well-being of healthcare workers? Applied Ergonomics, London, v. 39, n. 5, p. 589-596, 2008. http://dx.doi.org/10.1016/j. apergo.2008.02.019. PMid:18423559

BASTIEN, C. H.; VALLIÈRES, A.; MORIN, C. M. Validation of the Insomnia Severity Index as an outcome measure for insomnia research. Sleep Medicine, Amsterdam, v. 2, n. 4, p. 297-307, 2001. http://dx.doi.org/10.1016/S1389-9457(00)00065-4. PMid:11438246

BENEDITO-SILVA, A. A. et al. A self-assessment questionnaire for the determination of morningnesseveningness types in Brazil. Progress in Clinical and Biological Research, New York, v. 341B, p. 89-98, 1990. PMid:2217379.

BENVEGNÚ, L. A. et al. Prevalência de hipertensão arterial entre motoristas de ônibus em Santa Maria, Rio Grande do Sul. Revista Brasileira de Saúde Ocupacional, São Paulo, v. 33, n. 118, p. 32-39, 2008. http://dx.doi.org/10.1590/S0303-76572008000200004.

BUYSSE, D. J. et al. Can an improvement in sleep positively impact on health? Sleep Medicine Reviews, London, v. 14, n. 6, p. 405-410, 2010. http://dx.doi. org/10.1016/j.smrv.2010.02.001. PMid:20427212

CARSKADON, M. A.; DEMENT, W. C. Normal human sleep: an overview. In: KRYGER, M. H.; ROTH, T.;
DEMENT, W. C. (Ed.). Principles and practice of sleep disorders medicine. Philadelphia: W.B. Saunders, 1994. p. 16-25.

CEOLIM, M. F.; MENNA-BARRETO, L. Sleep/wake cycle and physical activity in healthy elderly people. Sleep Research Online, Los Angeles, v. 3, n. 3, p. 8795, 2000. PMid:11382906.

CHIU, M. C. et al. Evaluating work ability and quality of life for clinical nurses in Taiwan. Nursing Outlook, New York, v. 55, n. 6, p. 318-326, 2007. http://dx.doi. org/10.1016/j.outlook.2007.07.002. PMid:18061017

CICONELLI, R. et al. Tradução para a língua portuguesa e validação do questionário genérico de avaliação de qualidade de vida SF-36 (Brasil SF-36). Revista Brasileira de Reumatologia, São Paulo, v. 39, n. 3, p. 143-150, 1999.

COSTA, G. Shift work and health: current problems and preventive actions. Safety and Health at Work, Korea, v. 1, n. 2, p. 112-123, 2010. http://dx.doi. org/10.5491/SHAW.2010.1.2.112. PMid:22953171

CRISPIM, C. A. et al. The influence of sleep and sleep loss upon food intake and metabolism. Nutrition Research Reviews, Cambridge, v. 20, n. 2, p. 195-212, 2007. http://dx.doi.org/10.1017/S0954422407810651. PMid:19079870

FOLKARD, S.; TUCKER, P. Shift work, safety and productivity. Occupational Medicine, London, v. 53, n. 2, p. 95-101, 2003. http://dx.doi.org/10.1093/occmed/ kqg047. PMid:12637593

GRANDNER, M. A. et al. Problems associated with short sleep: bridging the gap between laboratory and epidemiological studies. Sleep Medicine Reviews, London, v. 14, n. 4, p. 239-247, 2010. http://dx.doi. org/10.1016/j.smrv.2009.08.001. PMid:19896872

GURUBHAGAVATULA, I. Consequences of obstructive sleep apnoea. The Indian Journal of Medical Research, New Delhi, v. 131, p. 188-195, 2010. PMid:20308744.

HÄRMÄ, M. et al. The effect of an irregular shift system on sleepiness at work in train drivers and railway traffic controllers. Journal of Sleep Research, Oxford, v. 11, n. 2, p. 141-151, 2002. http://dx.doi.org/10.1046/j.1365-2869.2002.00294.x. PMid:12028479

HORNE, J. A.; OSTBERG, O. A self-assessment questionnaire to determine morningness-eveningness in human circadian rhythms. International Journal of Chronobiology, London, v. 4, n. 2, p. 97-110, 1976. PMid:1027738.

HOWARD, M. E. et al. Sleepiness, sleep-disordered breathing, and accident risk factors in commercial vehicle drivers. American Journal of Respiratory and 
Critical Care Medicine, New York, v. 170, n. 9, p. 10141021, 2004. http://dx.doi.org/10.1164/rccm.2003121782OC. PMid:15317672

IBER, C. et al. The AASM manual for the scoring of sleep and associated events: rules, terminology and technical specifications. Westchester: American Academy of Sleep Medicine, 2007.

ILMARINEN, J.; TUOMI, K.; KLOCKARS, M. Changes in the work ability of active employees over an 11-year period. Scandinavian Journal of Work, Environment \& Health, Helsinki, v. 23, n. 1, p. 49-57, 1997. Supplement 1.PMid:9247995.

JASPER, H. H. The ten twenty electrode system of the international federation. Eletroencephalography and Clinical Neurophysiology, Netherlands, v. 110, p. 371375, 1958.

JOHNS, M. W. A new method for measuring daytime sleepiness: the Epworth sleepiness scale. Sleep, New York, v. 14, n. 6, p. 540-545, 1991. PMid:1798888.

KAGEYAMA, T. et al. Associations of sleep problems and recent life events with smoking behaviors among female staff nurses in Japanese hospitals. Industrial Health, Kawasaki, v. 43, n. 1, p. 133-141, 2005. http:// dx.doi.org/10.2486/indhealth.43.133. PMid:15732316

KOYAMA, R. G. et al. Prevalence of and risk factors for obstructive sleep apnea syndrome in Brazilian railroad workers. Sleep Medicine, Amsterdam, v. 13, n. 8, p. 1028-1032, 2012. http://dx.doi.org/10.1016/j. sleep.2012.06.017. PMid:22841037

KRIPKE, D. F. et al. Circadian phase response curves to light in older and young women and men. Journal of Circadian Rhythms, London, v. 5, n. 4, p. 4, 2007. http://dx.doi.org/10.1186/1740-3391-5-4. PMid:17623102

KU, C. H.; SMITH, M. J. Organisational factors and scheduling in locomotive engineers and conductors: effects on fatigue, health and social well-being. Applied Ergonomics, Oxford, v. 41, n. 1, p. 62-71, 2010. http://dx.doi.org/10.1016/j.apergo.2009.04.006. PMid:19447381

LOPES, C. et al. Relationship between the quality of life and the severity of obstructive sleep apnea syndrome. Brazilian Journal of Medical and Biological Research, São Paulo, v. 41, n. 10, p. 908-913, 2008. http://dx.doi.org/10.1590/S0100-879X2008005000036. PMid:18820762

MANNARINO, M. R.; DI FILIPPO, F.; PIRRO, M. Obstructive sleep apnea syndrome. European Journal of Internal Medicine, Basingstoke, v. 23, n. 7, p. 586-593, 2012. http://dx.doi.org/10.1016/j. ejim.2012.05.013. PMid:22939801

METZNER, R. J.; FISCHER, F. M. Fadiga e capacidade para o trabalho em turnos fixos de doze horas. Revista de Saude Publica, São Paulo, v. 35, n. 6, p.
548-553, 2001. http://dx.doi.org/10.1590/S003489102001000600008.

MILOSEVIC, M.et al. Work ability as a major determinant of clinical nurses' quality of life. Journal of Clinical Nursing, Oxford, v. 20, n. 19-20, p. 2931-2938, 2011. http://dx.doi.org/10.1111/j.13652702.2011.03703.x. PMid:21323781

MINA, R.; CASOLIN, A. National standard for health assessment of rail safety workers: the first year. Medical Journal of Australia, Sydney, v. 187, n. 7, p. 394-397, 2007. PMid:17908002.

MORGAN, L. et al. Circadian aspects of postprandial metabolism. Chronobiology International, London, v. 20, n. 5, p. 795-808, 2003. http://dx.doi.org/10.1081/ CBI-120024218. PMid:14535354

NEDELTCHEVA, A. V. et al. Insufficient sleep undermines dietary efforts to reduce adiposity. Annals of Internal Medicine, Philadelphia, v. 153, n. 7, p. 435441, 2010. http://dx.doi.org/10.7326/0003-4819-153-7201010050-00006. PMid:20921542

NENA, E. et al. Sleep-disordered breathing and quality of life of railway drivers in Greece. Chest, Chicago, v. 134, n. 1, p. 79-86, 2008. http://dx.doi. org/10.1378/chest.07-2849. PMid:18347205

OHAYON, M. M.; SMOLENSKY, M. H.; ROTH, T. Consequences of shiftworking on sleep duration, sleepiness, and sleep attacks. Chronobiology International, London, v. 27, n. 3, p. 575-589, 2010. http://dx.doi.org/10.3109/07420521003749956. PMid:20524802

OLIVEIRA E SILVA, L. et al. Mood, sleep patterns and the effect of physical activity on the life quality of brazilian train operators. Sleep Science, São Paulo, v. 5, n. 4, p. 113-119, 2012.

PADILHA, H. G. et al. Metabolic responses on the early shift. Chronobiology International, London, v. 27, n. 5, p. 1080-1092, 2010. http://dx.doi.org/10.3109/074 20528.2010.489883. PMid:20636217

RECHTSCHAFFEN, A.; KALES, A. A manual of standardized terminology, techniques, and scoring system for sleep stages of human subjects. Washington: Public Health Service, U.S. Government Printing Office, 1968.

RICHARDSON, D. et al. Time-related aspects of the healthy worker survivor effect. Annals of Epidemiology, New York, v. 14, n. 9, p. 633-639, 2004. http://dx.doi.org/10.1016/j.annepidem.2003.09.019. PMid:15380793

SALLINEN, M. et al. Sleep-wake rhythm in an irregular shift system. Journal of Sleep Research, Oxford, v. 12, n. 2, p. 103-112, 2003. http:// dx.doi.org/10.1046/j.1365-2869.2003.00346.x. PMid:12753347 
SPIEGEL, K. et al. Leptin levels are dependent on sleep duration: relationships with sympathovagal balance, carbohydrate regulation, cortisol, and thyrotropin. The Journal of Clinical Endocrinology and Metabolism, Springfield, v. 89, n. 11, p. 57625771, 2004. http://dx.doi.org/10.1210/jc.2004-1003. PMid:15531540

TUFIK, S. et al. Obstructive sleep apnea syndrome in the Sao Paulo Epidemiologic Sleep Study. Sleep Medicine, Amsterdam, v. 11, n. 5, p. 441-446, 2010. http://dx.doi.org/10.1016/j.sleep.2009.10.005. PMid:20362502

TUOMI, K. et al. Aging, work, life-style and work ability among Finnish municipal workers in 19811992. Scandinavian Journal of Work Environment
$\mathcal{E}$ Health, Helsinki, v. 23, n. 1, p. 58-65, 1997. Supplement 1. PMid:9247996.

TUOMI, K. et al. Índice de capacidade para o trabalho. São Carlos: EdUFSCar, 2005.

WAGSTAFF, A. S.; SIGSTAD LIE, J. A. Shift and night work and long working hours: a systematic review of safety implications. Scandinavian Journal of Work, Environment \& Health, Helsinki, v. 37, n. 3, p. 173-185, 2011. http://dx.doi.org/10.5271/sjweh.3146. PMid:21290083

ZHANG, L. et al. Cigarette smoking and nocturnal sleep architecture. American Journal of Epidemiology, Baltimore, v. 164, n. 6, p. 529-537, 2006. http://dx.doi. org/10.1093/aje/kwj231. PMid:16829553 